\title{
Diagnosis of Bacterial Vaginosis by Acridine Orange Staining and its Comparison to Conventional Methods and Association of Gardnerella vaginalis with Bacterial Vaginosis.
}

\author{
Naznin Begum ${ }^{1}$, Naima Muazzam², S.M Shamsuzzaman ${ }^{3}$, Akhtaruzzaman Chowdhury ${ }^{4}$, Aliya Rashid ${ }^{5}$, Dinul Islam ${ }^{6}$ \\ ${ }^{1,6}$ Department of Microbiology, Faridpur Medical College, Faridpur, ${ }^{2}$ Department of Microbiology, National Institute of \\ Preventive and Social Medicine (NIPSOM), Mohakhali, Dhaka, ${ }^{3-5}$ Department of Microbiology, Dhaka Medical College, Dhaka
}

\begin{abstract}
The present study was undertaken to establish the efficacy of acridine orange staining for diagnosis of Bacterial Vaginosis (BV) and association of Gardnerella Vaginalis with BV. Two hundred sexually active females in the age group of 15-45 years, with vaginal discharge and itching, were selected for the study. A detailed history and a thorough clinical examination of all the cases were done. After making the clinical diagnosis of BV by Amsels criteria, diagnosis also carried out with Acridine orange staining, Gram stain, Nugent criteria and isolation of Gard. vaginalis from vaginal fluid. Out of 200 women, $48(24 \%)$ cases were diagnosed as having bacterial vaginosis by applying Amsel's clinical criteria. The rate of detection of bacterial vaginosis was $23 \%$ by Gram stain Nugent criteria and $24.5 \%$ by acridine orange staining. Out of the total $48 \mathrm{BV}$ cases, the rate of detection of BV was $100 \%$ by Acridine orange staining and $93.87 \%$ by Gram-stain Nugent criteria. By Acridine orange staining Lactobacillus and pus cell were also detected which provided an important information about vaginal ecosystem. Acridine orange staining was the most sensitive (100\%) method considering Amsel's criteria as gold standard. The sensitivity of Gram stain Nugent criteria was $\mathbf{9 3 . 7 5 \%}$ in this study. The specificity of Acridine orange staining was $\mathbf{9 9 . 5 5 \%}$ and the specificity of Gram stain Nugent criteria was $99.10 \%$. The positive predicative values of acridine orange staining and Gram staining Nugent criteria were $97.96 \%$ and $95.74 \%$ and the negative predictive values of these tests were $100 \%$ and $98.65 \%$ respectively. The results of Acridine orange staining test correlated well with that of Amsel's clinical criteria among the study cases and healthy controls.
\end{abstract}

Key words: Bacterial Vaginosis, Sexually Transmitted Diseases, Amsel's criteria, Acridine orange, Nugent criteria, Gardnerella vaginalis.

\section{Introduction}

Bacterial vaginosis (BV) is the most common cause of abnormal vaginal discharge and one of the most prevalent lower genital tract infection affecting almost one third (29\%) of the women of reproductive age. ${ }^{1}$ The $\mathrm{BV}$ is a clinical condition characterized by a thin, gray, homogenous,

Correspondence:

Dr. Naznin Begum

Lecturer

Department of Microbiology

Faridpur Medical College, Faridpur, Bangladesh.

Email: drnaznin.@yahoo.com malodorous adherent vaginal discharge having $\mathrm{P}^{\mathrm{H}}>4.5$, release of a fishy odour on addition of $10 \% \mathrm{KOH}$ to the vaginal fluid (whiff test), presence of clue cell and a few or no Lactobacilli. ${ }^{2}$

The normal flora of vagina, Lactobacillus, which under physiologic condition, produces an acidic milieu by transforming glycogen into lactic acid through hydrogen per oxide production, this lactic acid suppresses the growth of other organisms. Change in the normal vaginal flora causes change in $\mathrm{p}^{\mathrm{H}}$ which allows $\mathrm{BV}$ associated organisms like Gardnerella vaginalis and other anaerobes to overgrow and cause chronic infection and discharge. ${ }^{3}$ Symptom of BV depends on concentration of Gardnerella vaginalis. ${ }^{4}$ Almost 
$50 \%$ of $\mathrm{BV}$ patients remain asymptomatic. Although symptoms occur, the manifestation of BV is mild, so usually overlooked in developing countries like Bangladesh. Diagnosis of BV is important for its serious complications such as premature rupture of membrane, miscarriage, development of pelvic inflammatory diseases, increase risk of acquiring STD such as HIV and also increase genital tract HIV shedding. ${ }^{5}$

The predominant organism that causes BV are Gardnerella vaginalis, Mycoplasma hominis and Ureaplasma urealiticum. Other anaerobes such as Prevotella, Mobiluncus, Bacteroides and Peptostreptococcus have also been identified as flora associated with BV. ${ }^{6}$ Recently discovered strict anaerobe Atopobium vaginae is another organism that is strongly associated with $\mathrm{BV}$, but its role in $\mathrm{BV}$ is still unknown. In women with BV, Gardnerella vaginalis is almost universally present $(99 \%)$ and majority have $A$. vaginae $(96 \%)$ infection. ${ }^{7}$ Gardnerella vaginalis has been reported to be present in small numbers $\left(<10^{4} \mathrm{cfu} / \mathrm{ml}\right)$ in asymptomatic women and its count of $\geq 10^{6} \mathrm{cfu} / \mathrm{ml}$ have always been associated with symptom of $\mathrm{BV}{ }^{8}$

The optimal method of diagnosing BV remains controversial. There are two main categories of diagnostic tests for BV: clinical criteria and laboratory based testing ${ }^{5}$. The most widely accepted clinical criteria are Amsel's clinical criteria. This clinical criteria require 3 of the following 4 signs such as vaginal fluid $\mathrm{P}^{\mathrm{H}}>4.5,>20 \%$ epithelial cells are clue cell, specific characteristic vaginal discharge of $\mathrm{BV}$ and a positive whiff test. Evaluation of the clinical parameters are more subjective and vary with the skill of the clinician. ${ }^{9}$

For laboratory testing Gram stain of vaginal smear was standardized by Nugent score. The result of Nugent score varies from person to person specially when different microscopes are being used. Culture of vaginal fluid is often used as a primary laboratory test in the past but this is found to be of little value in the diagnosis of BV as Gardnerella vaginalis is isolated in $83 \%$ to $94 \%$ of women with sign of BV but is also recovered in $36 \%$ to $55 \%$ of healthy women, as it is a normal flora of the human vagina. Oligonucleotide probe test for detection of Gardnerella vaginalis, detection of amine and fatty acid by electrophoresis and gas liquid chromatography, proline aminopeptidase and sialidase assay etc. are available. ${ }^{10}$ Among other laboratory methods, ELISA for detection of anti-hemolysin antibody of Gardnerella vaginalis, PCR for detection of $\mathrm{BV}$ associated bacteria, Affirm VP III microbial identification system, Quick Vue R advanced $\mathrm{p}^{\mathrm{H}}$ and amine test card for detection of $\mathrm{BV}$ are available in the advanced countries and these tests are restricted in the research laboratory. $7,11,12,13$

The use of acridine orange stain for detection of clue cells is shown to be very much effective for diagnosis of BV.
Acridine orange differentially stains the micro-organisms from cellular materials. Acridine orange staining technique has been recommended for the rapid identification of clue cells in vaginal smears. By acridine orange stain clue cell (orange staining bacteria on green epithelial cell) is clearly seen, number of Lactobacilli and pus cell can be observed. Other two common vaginal pathogens, Candida and Trichomonas can also be detected by this method. ${ }^{14}$

Diagnosis of BV by Amsel's clinical criteria and Gram stain Nugent criteria is not yet practiced in clinical laboratories in Bangladesh as it is time consuming and laborious. Diagnosis of $\mathrm{BV}$ is still based on only detection of clue cell by wet microscopy and Gram stain method.

Therefore the present study has been designed to establish an easy, simple, relatively inexpensive test for early diagnosis of bacterial vaginosis by acridine orange staining test which can help in proper diagnosis and treatment of $\mathrm{BV}$ and prevention of its complications.

\section{Methods}

This was a prospective study carried out in the department of Microbiology, Dhaka Medical College. Two hundred patients attending Gynae outpatient department of Dhaka Medical College and Maternal and Child Health Training Institute, Azimpur, Dhaka with history of abnormal characterstic vaginal discharge and itching suspected to have BV were included in the study.

Under all aseptic precaution, vaginal examination was done. At the time of speculum examination, the presence of vaginal discharge was noted, the vaginal $\mathrm{PH}^{\mathrm{H}}$ was recorded and amine test was done.

(i) Physical examination of discharge:

Specific characteristic vaginal discharge of BV patient was noted which was thin, gray/white, homogenous, foul smelling (fishy odor) and adherent to the vaginal wall.

(ii) Measurement of vaginal fluid $\mathrm{P}^{\mathrm{H}}$ :

$\mathrm{P}^{\mathrm{H}}$ was measured by placing a $\mathrm{P}^{\mathrm{H}}$ paper (Merck, UK) by sponge holding forceps into the vagina during vaginal examination and colour change was observed. When the $\mathrm{P}^{\mathrm{H}}$ of vaginal fluid was $>4.5$, the cases were suspected as BV cases.

(iii) Amine test (Whiff test):

One drop of $10 \% \mathrm{KOH}$ was added with vaginal discharge and immediately sniffed for the fishy odor.

(iv) Wet Mount preparation:

One drop of normal saline was taken on a glass slide and vaginal discharge was added and covered with a cover slip. Then the slide was examined under light microscope. 
$\mathrm{BV}$ was dignosed by the presence of clue cell in association with at least 2 of the other 3 Amsel's clinical criteria.

Diagnosis of bacterial vaginosis by Gram stain Nugent criteria $^{15}$ :

Each Gram stained smear was evaluated for the following morphotypes under oil immersion ( $\times 1000$ magnification) according to the Nugent criteria.

Acridine orange test ${ }^{14}$ :

The unfixed dried smear was stained with the Acridine orange acid staining and microscopic examination was done initially with $10 \times$ objective to see the distribution of fluorescing material, and then with 40×objective to identify T. vaginalis, yeast cells, clue cell and pus cell.

Identification of different structures in acridine orange staining:

Normal epithelial cells were stained pale green colour with green coloured nucleus. Lactobacillus stained green colour.

Gardnerella vaginalis stained yellowish orange.

Leucocytes (pus cells)-yellowgreen.

Trichomonas. vaginalis was identified by orange red staining with yellow-green nucleus.

Yeast cells were stained-orange colour.

In bacterial vaginosis, the orange staining bacteria adhered to the green epithelial cells were clue cells.

Inflammatory cell stained yellowish green with yellow to orange coloured nucleus.

Culture for isolation of Gardnerella vaginalis ${ }^{16}$ :

Human blood bilayer Tween 80 (HBT) agar media was inoculated directly from swab sample and was incubated in the candle extinction jar containing water-soaked cotton at $37^{\circ} \mathrm{C}$ for $48-72$ hours for primary isolation of Gard. vaginalis. The plates were examined by oblique lighting after $24 \mathrm{hrs}$., $48 \mathrm{hrs}$. and $72 \mathrm{hrs}$.

Then semi quantitative estimation of Gardnerella vaginalis in culture was done.

The number of colonies were counted and expressed as $1+$ to $4+$ according to the colonies found in different streaking zone.

Growth was quantitated as follows:

$1+,<10$ colonies in the first streaking zone;

$2+,>10$ colonies in the first zone and,$<10$ colonies in the $2^{\text {nd }}$ zone;

$3+,>10$ colonies in the $2^{\text {nd }}$ zone and $<10$ colones in the $3^{\text {rd }}$ zone;

$4+,>10$ colonies in the $3^{\text {rd }}$ zone.

Identification of Gardnerella vaginalis ${ }^{17,18}$

i. Colonial morphology: Colonies on HBT agar were identified as small white colonies with $\beta$-hemolysis after 48 or 72 hours of incubation.

ii. Clear $\beta$-hemolysis with diffuse edges on HBT media, but no hemolysis on sheep blood agar.

iii. The typical cell morphology in a Gram stained smear from a colony were pleomorphic, Gram-variable or Gram-negative coccobacilli or small rods.

iv. Catalase test negative.

v. Oxidase test negative.

vi. Susceptibility to different antimicrobial agents: 1 . Bacitracin (5IU) sensitive, 2. Metronidazole $(5 \mu \mathrm{g})$ resistance, 3. Metronidazole $(50 \mu \mathrm{g})$ sensitive, 4 . Sulphonamide $(100 \mu \mathrm{g})$ resistance, 5 . Nitrofurantoin $(150 \mu \mathrm{g})$ sensitive

\section{Results}

All BV cases were diagnosed using the presence of clue cells' and two or more of the other three Amsel's criteria.

Of the 200 patients, 48 (24\%) were positive for BV by Amsel's clinical criteria, 46 (23\%) patients were positive for BV by Gram stain Nugent criteria $49(24.5 \%)$ were positive by acridine orange staining and $51(25.5 \%)$ were culture positive for G. vaginalis (Table 1).

Out of 49 acridine orange staining positive patients, 48 were positive by Amsel's criteria and one was negative. All the patients who were positive by Amsel's clinical criteria were positive by acridine orange staining. Out of 46 cases detected by Gram stain Nugent criteria, one was negative by Amsel's clinical criteria. Amsel's criteria detected additional 3 cases of BV who were negative by Gram stain Nugent criteria, Of the 51 women who were positive for Gard. vaginalis by culture, 5 were negative by Amsel's criteria.

Table 1: Comparison of Amsel's criteria with Gram stain Nugent criteria, acridine orange staining and culture of vaginal fluid for Gard. vaginalis for diagnosis of BV.

\begin{tabular}{lllllll}
\hline $\begin{array}{l}\text { BV diagnosis } \\
\text { by Amsel's criteria }\end{array}$ & \multicolumn{2}{c}{$\begin{array}{c}\text { Gram stain } \\
\text { Nugent criteria } \\
\text { Positive }\end{array}$} & $\begin{array}{r}\text { Negative } \\
\text { Posidine orange } \\
\text { Staining }\end{array}$ & $\begin{array}{c}\text { Culture for } \\
\text { G. vaginalis } \\
\text { Pegative }\end{array}$ & $\begin{array}{c}\text { Positive } \\
\text { Negative }\end{array}$ \\
\hline $\begin{array}{l}\text { BV cases } \\
(\mathrm{n}=48)\end{array}$ & $45(93.75)$ & $3(6.25)$ & $48(100.00)$ & $0(0.00)$ & $46(95.83) 2(4.17)$ \\
$\begin{array}{l}\text { Non BV } \\
(\mathrm{n}=152)\end{array}$ & $1(0.66)$ & $151(99.34)$ & $1(0.66)$ & $151(99.34)$ & $5(3.29)$ & $147(96.71)$ \\
Total 200 & 46 & 154 & 49 & 151 & 51 & 149 \\
\hline
\end{tabular}

Figures in parentheses represent percentages.

Gram staining, value $=173.29, \mathrm{df}=1, \mathrm{P}$ value $=<0.001 * * *$

Acridine orange staining, value $=189.30, \mathrm{df}=1, \mathrm{P}$ value $=<$ $0.001^{* * *}$

Culture, value $=159.62, \mathrm{df}=1, \mathrm{P}$ value $=<0.001^{* * *}$

Note: $* * *$ highly significant 
Among the BV cases diagnosed by different methods, 46 (95.83\%) of the 48 cases were diagnosed by Amsel's criteria, $43(93.48 \%)$ of the 46 cases by Gram stain Nugent criteria and $47(95.92 \%)$ of the 49 cases by Acridine orange staining were positive for G. vaginalis in culture (Table-2).

Table 2: Isolation of G. vaginalis among BV cases diagnosed by different methods.

\begin{tabular}{lccc}
\hline $\begin{array}{l}\text { Culture } \\
\text { for }\end{array}$ & $\begin{array}{l}\text { Amsel's } \\
\text { criteria } \\
(\mathrm{n}=48)\end{array}$ & $\begin{array}{c}\text { BV positive cases } \\
\text { Gram stain Nugent criteria } \\
(\mathrm{n}=46)\end{array}$ & $\begin{array}{c}\text { Acridine orange stain } \\
(\mathrm{n}=49)\end{array}$ \\
\hline Positive & $46(95.83)$ & $43(93.48)$ & $47(95.92)$ \\
Negative & $2(4.17)$ & $3(6.52)$ & $2(4.08)$ \\
\hline
\end{tabular}

Figures in parentheses represent percentages.

In wet film examination, $51(25.5 \%)$ were positive for clue cell, $16(8 \%)$ were positive for Trichomonas vaginalis and 62 $(31 \%)$ were positive for Candida. In 11 cases, both clue cell and Trichomonas vaginalis were found. By Gram staining method, $49(24.5 \%)$ were positive for clue cell, $9(4.5 \%)$ were positive for Trichomonas vaginalis and 48 (24\%) were positive for Candida. By Acridine orange staining 49 (24.5\%) were positive for clue cell, $22(11 \%)$ were positive for Trichomonas vaginalis and $69(34.50 \%)$ were positive for Candida (Table-3).

Table 3: Detection of clue cell, Trichomonas vaginalis and Candida by wet film examination, Gram staining \& acridine orange staining of vaginal fluid among the study cases.

\begin{tabular}{llll}
\hline $\begin{array}{l}\text { Vaginal } \\
\text { infection }\end{array}$ & $\begin{array}{l}\text { Wet film } \\
\text { examination } \\
\text { Positive }\end{array}$ & $\begin{array}{l}\text { Gram } \\
\text { staining } \\
\text { Positive }\end{array}$ & $\begin{array}{l}\text { Acridine orange } \\
\text { staining } \\
\text { Positive }\end{array}$ \\
\hline Clue cell & $40+11^{*=51(25.50)}$ & $49(24.50)$ & $49(24.50)$ \\
Trichomoniasis & $5+11^{*=16(8.00)}$ & $9(4.50)$ & $22(11.00)$ \\
Candidiasis & $62(31.00)$ & $48(24.00)$ & $69(34.50)$ \\
\hline
\end{tabular}

Figures in parentheses represent percentages.

*11 patients had both trichomoniasis and bacterial vaginosis.

Clue cells were found in 51 cases by wet film microscopy, and of them, BV was diagnosed in 48 cases by Amsel's clinical criteria. Gram stain method detected 49 clue cell positive cases, 46 of them were BV positive by Nugent criteria. Acridine orange staining detected 49 clue cell positive cases and all were BV positive (Table-4).
Table 4: Bacterial vaginosis cases among the women who were positive for clue cells by different methods.

\begin{tabular}{lcc}
\hline Methods & clue cell positive & BV positive \\
\hline Wet film & 51 & $* 48(94.11 \%)$ \\
Gram staining & 49 & $* * 46(93.87 \%)$ \\
Acridine orange staining & 49 & $* * * 49(100.00 \%)$ \\
\hline
\end{tabular}

Note:

* BV diagnosed by Amsel's criteria,

** BV diagnosed by Gram stain Nugent criteria,

*** BV diagnosed by only Acridine orange staining.

\section{Discussion:}

In this study, out of 200 study cases, 49 were diagnosed as $\mathrm{BV}$ by Acridine orange technique. Only one case from the control group was diagnosed as BV by Acridine orange stain who was found negative for BV by other methods.

Amsel's clinical criteria detected 48 cases of BV and all those 48 cases were also found positive by Acridine orange method. The Gram-stain Nugent criteria detected 46 BV cases, out of them 45 were positive by Amsel's clinical criteria. Acridine orange staining detected 3 cases of $\mathrm{BV}$ who were also positive by Amsel's criteria but were negative by Gram stain Nugent criteria. Acridine orange test also detected one clue cell positive women from study cases who was negative by Amsel's clinical criteria. This might be due to the fact that the women were clue cell positive by smear but result of $\mathrm{p}^{\mathrm{H}}$, amine test were altered by tropical ointment, blood or semen. Culture of vaginal fluid for $G$. vaginalis revealed 46 culture positive cases among $48 \mathrm{BV}$ cases. Culture was also positive in 5 cases among the non $\mathrm{BV}$ groups for $G$. vaginalis. G. vaginalis may be present as normal flora of the vagina. So the presence of $G$. vaginalis does not always indicate that the patient is suffering from BV. But in $\mathrm{BV}$, number of $G$. vaginalis is increased.

In order to determine the pathogenic role of $G$. vaginalis in $\mathrm{BV}$, quantitative estimation of $G$. vaginalis in culture has been recommended. In this study, most of the BV cases yielded $3+$ to $4+$ growth of $G$. vaginalis in culture but non BV cases and healthy controls yielded $1+$ or $2+$ growth.

In this study, wet film microscopy detected $51(25.5 \%)$ clue cell, 16(8\%) Trichomonas vaginalis and 62 (31\%) Candida respectively. Gram stain method detected $49(24.50 \%)$ clue cell, 9(4.5\%) Trichomonas vaginalis and 48(24\%) Candida. Acridine orange stain detected 49 clue cells, 22 Trichomonas vaginalis and 69 Candida. Among 51 clue cell positive cases (by wet film), 48 were positive for BV by Amsel's criteria. Of the 49 clue cell positive cases by Gram stain method, 45 women were categorized into BV group according to Gram stain Nugent criteria. By Acridine orange staining $49 \mathrm{BV}$ 
cases were diagnosed by observing clue cell, condition of Lactobacillus and pus cell. Out of them 48 were also BV positive by Amsel's clinical critieria. Wet film examination detected additional 3 clue cell positive cases who were BV negative by Amsel's criteria. Gram stain method failed to detect $3 \mathrm{BV}$ cases who were clue cell positive but negative by Nugent criteria. Amsel's criteria also missed one case who was BV positive by acridine orange staining. So further study is required with molecular method to confirm whether the case was false positive or not. Moreover, for diagnosis of BV cases by Amsel's criteria, only wet film microscopy is not sufficient. Presence of clue cell among the patients with BV ranges from 85 to 97 percent compared with 5 to 14 percent women without BV. For diagnosis of BV by clue cell in wet microscopy requires at least 20 fields examination and also additional 2 criteria which is time consuming and more subjective. On the other hand, BV can be diagnosed directly by examining the Acridine orange stained vaginal fluid smear. Moreover, wet film microscopy may over diagnose the BV cases. But by acridine orange staining method BV cases can be diagnosed accurately because by this technique clue cell, Lactobacillus and pus cell are better visualized. So Acridine orange staining is better than any other method.

The Acridine orange test detected highest number (49) of BV cases. In this study, acridine orange staining, Gram stain Nugent criteria and Amsel's criteria detected 49(100\%), $46(93.87 \%)$ and $48(94.11 \%)$ BV cases respectively. In contrast to the present study, Nunns et al. (1996) detected 63 (77.81\%), 40 (49.4\%) and 48(59.3\%) BV cases by Acridine orange staining, Gram stain Nugent criteria and Amsel's criteria respectively.

The Gram stain Nugent criteria based on bacterial morphotype count is also laborious. The reliability of diagnosing BV by Gram stain method has been improved by a scoring system (0-10) of interpretation by Nugent. The 'intermediate' score group of Gram stain Nugent criteria has a higher $(32 \%)$ chance of transition to BV. Women of this group are more susceptible to acquisition of HIV and other STDs as they do not receive any treatment and thereby increased risk of complications of BV.

The results of Gram stain varies from person to person specially when different microscopes are used as the actual field diameter is not mentioned in the system. Moreover, sometimes Gram stain cannot differentiate very short form of Lactobacillus from Gardnerella vaginalis Clue cell can also be found in intermediate score group. The women of this group may develop BV which can be missed if only Gram stain Nugent score is done for diagnosis of BV.

The acridine orange test yielded excellent sensitivity and specificity with respect to Amsel's clinical criteria, Gramstain Nugent criteria and culture of $G$. vaginalis. The sensitivity was very high $(100 \%)$ within the range of acceptable values as a reliable diagnostic test, the specificity was also high (99.55\%) and acceptable.

\section{References}

1. Klebanoff MA, Schwebke JR, Zhang J, Nansel TR, Yu KF, Andrews WW. Vulvovaginal symptoms in women with bacterial vaginosis. Obstet Gynecol 2004;104:267-72.

2. Holmes KK, Spiegel C, Amsel R, Eschenbach DA, Chen KCS, Totten P. Nonspecific vaginosis. Scand J Infect Dis Suppl 1981; 26:110-4.

3. Ramani JT, Kavita R, Legori M. prevalence of bacterial vaginosis in women attendeing antenatal, Gynaecology and STD clinics of Medical College, Thiruvanthapuram. J Acad clin Microbiol. 2004; $1: 107-11$.

4. Greenwood JR, Pickett MJ, Martin WJ, Mack EG. Haemophilus vaginalis (Corynebacterium vaginale) : method for isolation and rapid biochemical identification. Health Lab Sci 1977; 14:102-6.

5. Sha B. Utility of Amsel's criteria, Nugent score and quantitative PCR for Gardnerella vaginalis, Mycoplasma hominis and Lactobacillus spp. for diagnosis of bacterial vaginosis in Human Immunodeficiency virus infected women. J Clin Microbiol. 2005; 43: 4607-4612.

6. Owen MK, Clenney TL. Management of vaginitis. Am Fam Physician 2004; 70:2125-32.

7. Bradshaw CS, Tabrizi SN, Faisley CK, Morton AN, Rudland E. Gardland SM. The association of Atopobium vaginae and Gardnerella vaginalis with bacterial vaginosis and recurrence after oral metronidazole therapy. J Infect Dis. 2006; 194: 828-36.

8. McCormack WM, Hayes L, Rosner B, Evrard JR, Crockett VA, Alpert S, Zinner SH. Vaginal colonization with Corynebacterium vaginale (Haemophilus vaginalis). J Infect Dis 1977; 136:740-5.

9. French L, Horton J, Matousek M. Abnormal vaginal discharge: what does and does not work in the underlying causes. J Fam Pract 2004; 53:890-4.

10. Hillier SL, Watts H, Hanssen WP, Holmes KK. The normal vaginal flora, $\mathrm{H}_{2} \mathrm{O}_{2}$ producing Lactobacilli and bacterial vaginosis in pregnant women. Clin Infect Dis 1993; 16:273-81.

11. Cauci S, Scrimin F, Driussi S, Ceccone S, Monte R, Fant L, et al. Specific immune response against Gard. vaginalis hemolysin in patients with bacterial vaginosis. Am J obstet Gynecol 1997; 175:1601-5.

12. Brown HL, Fuller DD, Jasper LT. Davis TE, Wright JD. Clinical evaluation of Affirm VPIII in the detection and identification of Trichomonas vaginalis, Gardnerella vaginalis, and Candida species in vaginitis/vaginosis. Infect Dis Obstet Gynecol 2004; 11: $12-7$. 
13. Soper DE. Taking the guesswork out of diagnosing and managing vaginitis. Obstet Gynecol 2005; 50:32-9.

14. Nunns D, Mandel D, Farrand RJ, O' Neill H, Henshaw G. A comparison of acridine orange, wet microscopy and Gram staining in the diagnosis of bacterial vaginosis. J Infect 1997; 34: 211-3.

15. Nugent RP, Krohn MA, Hiller SL. Reliability of diagnosing bacterial vaginosis is improved by a standardized method of Gram stain interpretation. J Clin Microbiol 1991; 29: 297-301.

16. Patricia A, Totten, Amsel R, Hale J, Piot P, Holmes KK. Selective differential human blood bilayer media for isolation of Gardnerella vaginalis. J Clin microbiol 1982; 15(1): 141-7.

17. Collee JG, Howard AF, Iscon CA. Haemophilus, Gardnerella and other bacilli. In: Collce JG, Fraser AG, Marmion BP, Simmons A, editors. Mackic and McCartney practical medical microbiology, $14^{\text {th }}$ edition, USA: Churchill Living stone Inc; 1996: pp. 456-9.

18. Piot P, Van dyck E, Godts P, Vanderheyden J. The vaginal microbial flora in nonspecific vaginitis. Eur J Clin Microbiol 1982; 1: 301-6. 